Набродова Е.Г. Шмигельский В.А. Сурнева О.Б. Яновская О.С.

Введение

Результаты исследования:

Обсуждение и заключение

Ключевые слова
Северо-Кавказский федеральный университет

Россия,

г. Ставрополь.

shmigelskiy.92777@mail.ru

\section{ИССЛЕДОВАНИЕ ЗАВИСИМОСТИ СКОРОСТИ РАСПРОСТРАНЕНИЕ ГРАВИТАЦИОННЫХ ВОЛН В АТМОСФЕРЕ ОТ ШИРОТЫ}

Волны в атмосфере играют важную роль, так как они определяют как интенсивность погодообразующих явлений, так и скорость их перемещения. Правда остается открытым вопрос о причине возбуждения планетарных волн. Они могут возбуждаться процессами, происходящими в нижней атмосфере, а также их причиной могут быть возмущения стратоссреры. В любом случае, сфрормировавшись, планетарная волна будет влиять на процессы, происходящие в тропоссрере. Поэтому исследование скорости их распространения является актуальной проблемой в задачах прогнозирования погоды.

Материалы и методы: Система уравнений, описывающих крупномасштабную динамику атмосферы, является сложной и в общем виде не решаема. Поэтому обращаются к процедуре линеаризации уравнений. Насколько это правомочно остается открытым, так как волны могут быть нелинейными и все разнообразие явлений обусловлено именно их нелинейностью. Рассмотрим движение сухого воздуха, описываемого уравнением движения идеальной жидкости в неинерциальной системе отсчета, с учетом вращения Земли:

$\frac{\partial \mathbf{v}}{\partial t}+(\mathbf{v}, \nabla) \mathbf{v}=\mathbf{g}-\frac{1}{\rho} \nabla p+2\left[\mathbf{v} \omega_{0}\right]$

Получилось сложное уравнение 4-го порядка. Рассмотрим предельные случаи: высокочастотные и низкочастотные колебания. В случае высокочастотных колебаний можно записать.

$$
\begin{aligned}
& \omega^{4}-\left(2 \omega_{0 z}\right)^{2} R \alpha\left(\gamma_{\mathrm{A}}-\gamma\right) \gamma k_{z}+\left(2 \omega_{0 z}\right)^{2} R \gamma k_{z}^{2}=0, \\
& \omega=(1 \pm i) \sqrt{\omega_{0 z}}\left(R \gamma k_{z}\right)^{1 / 4}=\omega_{\mathrm{r}} \pm i \omega_{\mathrm{i}}, \omega_{\mathrm{r}}=\sqrt{\omega_{0 z}}\left(R \gamma k_{z}\right)^{1 / 4}
\end{aligned}
$$

Таким образом, рассмотрев систему уравнений, описывающих динамику внутренних гравитационных волн в атмосфере, после линеаризации мы получили дисперсионное соотношение. Система уравнений решалась в приближении $f$-плоскости, т.е. параметр Кориолиса считался постоянным. При этом считалось, что плотность воздуха в состоянии статики атмоссреры подчиняется барометрическому закону. В пределах тропосферы такое допущение является приемлемым.

Внутренние гравитационные волны, уравнение движения, уравнение неразрывности, уравнение теплопроводности, статика атмоссреры, процедура линеаризации, дисперсионное соотношение, линейные волны. 
Nabrodova Ye.G., Shmigelsky V.A., Surneva O.B. Yanovskaya O.S.

Introduction:

Materials and methods:

Discussion and conclusion

Key words:
North-Caucasian Federal University, Stavropol,

Russia,

shmigelskiy.92777@mail.ru

\section{To the dependence of speed spray distribution of gravitational waves in the atmosphere of latitude}

Waves in the atmosphere play an important role, as they determine both the intensity of weather-forming phenomena and the speed of their movement. The truth remains the question of the reason for the excitation of planetary waves. They can be excited by processes occurring in the lower atmosphere, as well as their cause may be disturbances of the stratosphere. In any case, having formed, the planetary wave will influence the processes occurring in the troposphere. Therefore, the study of the speed of their distribution is a pressing problem in weather forecasting problems.

The system of equations describing the large-scale dynamics of the atmosphere is complex and cannot be solved in general. Therefore, refer to the procedure of linearization of equations. The extent to which it is valid remains open, since the waves can be non-linear and the whole variety of phenomena is determined precisely by their non-linearity.

Consider the movement of dry air, described by the equation of motion of an ideal fluid in a non-inertial reference system, taking into account the rotation of the Earth:

$$
\frac{\partial \mathbf{v}}{\partial t}+(\mathbf{v}, \nabla) \mathbf{v}=\mathbf{g}-\frac{1}{\rho} \nabla p+2\left[\mathbf{v \omega}_{0}\right]
$$

Results of the research: The result is a complex equation of the 4th order. Consider the limiting cases: high-frequency and low-frequency oscillations. In the case of high-frequency oscillations, we can write:

$$
\begin{aligned}
& \omega^{4}-\left(2 \omega_{0 z}\right)^{2} R \alpha\left(\gamma_{\mathrm{A}}-\gamma\right) \gamma k_{z}+\left(2 \omega_{0 z}\right)^{2} R \gamma k_{z}^{2}=0 \\
& \omega=(1 \pm i) \sqrt{\omega_{0 z}}\left(R \gamma k_{z}\right)^{1 / 4}=\omega_{\mathrm{r}} \pm i \omega_{\mathrm{i}} \\
& \omega_{\mathrm{r}}=\sqrt{\omega_{0 z}}\left(R \gamma k_{z}\right)^{1 / 4}
\end{aligned}
$$

Thus, having considered the system of equations describing the dynamics of internal gravity waves in the atmosphere, after linearization we obtained the dispersion relation. The system of equations was solved in the f-plane approximation, i.e. Coriolis parameter was considered constant. At the same time, it was believed that the density of air in the state of static atmosphere obeys the barometric law. Within the troposphere, this assumption is acceptable.

internal gravity waves, equation of motion, continuity equation, heat equation, atmospheric static, linearization procedure, dispersion relation, linear waves. 


\section{ВВЕДЕНИЕ}

Волны в атмосфере - это процесс распространения периодических движений, налагающихся на общий перенос воздуха [3]. Кроме упругих продольных звуковых (акустических) волн, в атмосфере существует несколько типов атмосферных волн, различных по происхождению и характеру с большими длинами волн. К таким волнам относятся волны, развивающиеся на границе двух воздушных слоёв, движущихся с разными скоростями и имеющими различные плотности и температуры. При этом в гребнях волн, где имеет место восходящее движение воздуха, происходит охлаждение воздуха, содержащийся в нём водяной пар конденсируется, и образуются облака. В ложбинах волн, где возникают нисходящие течения, воздух нагревается и удаляется от состояния насыщения, и небо между гребнями остается чистым, в результате появляются гряды волнистых облаков [1]. Аналогичный процесс происходит в так называемых горных волнах, возникающих при обтекании гор, возвышенностей и т.п. Колебательные движения продолжаются довольно долго после того, как данный объём воздуха миновал горное препятствие Волны этого типа - короткие волны - широко распространены. Они влияют на полёт летательных аппаратов, часто порождая, например, болтанку самолётов. Амплитуда и длина волн этого типа тем больше, чем больше разность скоростей движущихся масс и чем меньше разность плотностей и температур. Длина волн - от сотен метров до десятков километров, а амплитуда до 1-2 километров. Скорости восходящих движений, например, в гребнях горных волн могут достигать нескольких метров в секунду, этой их особенностью пользуются планеристы [2].

Кроме коротких волн в атмосфере (когда частицы колеблются в вертикальной плоскости), в атмосфере существуют волны крупного масштаба с длинами в сотни и тысячи километров; колебания в этом случае происходят преимущественно в горизонтальном направлении. Во-первых, это циклонические волны, возникающие на атмосферных фронтах, т.е. на границах между воздушными массами с разной температурой. При потере устойчивости эти волны приводят к образованию циклонов. Существуют также так называемые длинные волны: господствующий в средних широтах земной атмосферы западный поток является волнообразным; длина этих волн порядка нескольких тысяч километров, так что по окружности земного шара обычно укладывается несколько (3-6) длинных волн. Одна из причин их возникновения - различие в температурных условиях континентов и океанов. Циклонические и длинные волны в атмосфере определяют режим погоды над большими территориями; их изучение играет первостепенную роль для прогноза погоды [4].

Существуют и другие типы волн в атмосфере: волны тропопаузы - изменения высоты тропопаузы при перемещении в атмосфере циклонов и антициклонов; приливные волны, обусловленные притяжением Луны и Солнца [5]. 
В настоящей работе аналитическими методами получено дисперсионное соотношение для планетарных волн в атмосфере в приближении $\mathrm{f}$-плоскости и получено выражение для скорости их распространения в зависимости от широты.

\section{Материалы и методы исследования}

Рассмотрим движение сухого воздуха, описываемого уравнением движения идеальной жидкости в неинерциальной системе отсчета, с учетом вращения Земли [1-10]:

$$
\frac{\partial \mathbf{v}}{\partial t}+(\mathbf{v}, \nabla) \mathbf{v}=\mathbf{g}-\frac{1}{\rho} \nabla p+2\left[\mathbf{v} \omega_{0}\right]
$$
где $\mathbf{v}-\quad$ скорость движения частицы воздуха;
$\rho-\quad$ плотность движущейся частицы воздуха;
$p$ - $\quad$ давление на движущуюся частицу воздуха со стороны ок- ружающего ее воздуха.

Рассмотрим плоскость, касательную к поверхности Земли в данной точке. Ось $z$ направим перпендикулярно поверхности Земли. В состоянии равновесия (статики):

$$
\mathbf{v}=0, \quad \mathbf{g}-\frac{1}{\rho_{\mathrm{e}}} \nabla \bar{p}=0 .
$$

Здесь $\rho_{\mathrm{e}}-\quad$ плотность воздуха в состоянии статики;

$$
g-\quad \text { ускорение свободного падения. }
$$

Давление можно представить в виде $p=\bar{p}+p^{\prime}$. Параметры атмосферы в состоянии статики мы рассматриваем как невозмущенное состояние: $\rho=\rho+\rho^{\prime}$,

$$
\rho_{\mathrm{e}}(z)=\rho_{\mathrm{e} 0} e^{-\alpha\left(\gamma_{\mathrm{A}}-\gamma\right)(z+h)}
$$
- $\quad$ распределение плотности воздуха с высотой в состоянии статики атмосферы;
$\gamma_{\mathrm{A}}-\quad$ так называемый, градиент автоконвекиции, он совпадает с градиентом температуры однородной по плотности атмос- феры.

Запишем уравнение неразрывности в декартовой системе координат 


$$
\frac{\partial \rho^{\prime}}{\partial t}+u \frac{\partial \rho^{\prime}}{\partial x} \rho+v \frac{\partial \rho^{\prime}}{\partial y}+w \frac{\partial \rho^{\prime}}{\partial z}+w \frac{\partial \rho_{\mathrm{e}}}{\partial z}+\left(\frac{\partial u}{\partial x}+\frac{\partial v}{\partial y}+\frac{\partial w}{\partial z}\right)=0
$$

Будем считать, что температура атмосферы в состоянии статики изменяется по линейному закону: $T_{\text {e0 }}(z)=T_{\text {e0 }}-\gamma z$, где $\gamma$ - градиент температуры воздуха в состоянии статики; $T_{\text {е0 }}$ - температура воздуха вблизи поверхности земли в состоянии статики.

Будем считать, что температуру возмущенной атмосферы можно представить в виде:

$$
T=T_{\mathrm{e}}(z)+\theta(x, y, z)
$$

здесь $\theta(x, y, z)-$ ее возмущение, неизвестная функция.

\section{Уравнение теплопроводности:}

$$
\frac{\partial \theta}{\partial t}+(\mathbf{v}, \nabla) \theta=\gamma \cdot w+\kappa \nabla^{2} \theta
$$

где $к-$

коэффициент температуропроводности.

Если принять $\kappa=0$, что представляется разумным, так как мы рассматриваем движение невязкого воздуха (идеальная жидкость) с коэффициентом вязкости равным нулю $(v=0)$, то получим

$$
\frac{\partial \theta}{\partial t}+u \frac{\partial \theta}{\partial x}+v \frac{\partial \theta}{\partial y}+w \frac{\partial \theta}{\partial z}=\gamma \cdot w
$$

Таким образом, мы имеем систему уравнений

$$
\begin{aligned}
& \frac{\partial u}{\partial t}+u \frac{\partial u}{\partial x}+v \frac{\partial u}{\partial y}+w \frac{\partial u}{\partial z}=-\frac{1}{\rho_{\mathrm{e}}}\left(\frac{\partial p^{\prime}}{\partial x}\right)+2 w \omega_{0 z}-2 w \omega_{0 y}, \\
& \frac{\partial v}{\partial t}+u \frac{\partial v}{\partial x}+v \frac{\partial v}{\partial y}+w \frac{\partial v}{\partial z}=-\frac{1}{\rho_{\mathrm{e}}}\left(\frac{\partial p^{\prime}}{\partial y}\right)-2 \omega_{0 z} u \\
& \frac{\partial w}{\partial t}+u \frac{\partial w}{\partial x}+v \frac{\partial w}{\partial y}+w \frac{\partial w}{\partial z}=-\frac{1}{\rho_{\mathrm{e}}}\left(\frac{\partial p^{\prime}}{\partial z}\right)-g \rho^{\prime}+2 \omega_{0 y} u \\
& \frac{\partial \rho^{\prime}}{\partial t}+u \frac{\partial \rho^{\prime}}{\partial x}+v \frac{\partial \rho^{\prime}}{\partial y}+w \frac{\partial \rho^{\prime}}{\partial z}+w \frac{\partial \rho_{\mathrm{e}}}{\partial z}+\left(\frac{\partial u}{\partial x}+\frac{\partial v}{\partial y}+\frac{\partial w}{\partial z}\right)=0 \\
& \frac{\partial \theta}{\partial t}+u \frac{\partial \theta}{\partial x}+v \frac{\partial \theta}{\partial y}+w \frac{\partial \theta}{\partial z}=\gamma \cdot w .
\end{aligned}
$$




\section{Линеаризация уравнений \\ и дисперсионное соотношение}

Линеаризуем систему уравнений (7) - (11):

$$
\begin{aligned}
& \frac{\partial u}{\partial t}=-\frac{1}{\rho_{\mathrm{e}}}\left(\frac{\partial p^{\prime}}{\partial x}\right)+2 \tau \omega_{0 z}-2 w \omega_{0 y}, \\
& \frac{\partial v}{\partial t}=-\frac{1}{\rho_{\mathrm{e}}}\left(\frac{\partial p^{\prime}}{\partial y}\right)-2 \omega_{0 z} u, \\
& \frac{\partial w}{\partial t}=-\frac{1}{\rho_{\mathrm{e}}}\left(\frac{\partial p^{\prime}}{\partial z}\right)-g \rho^{\prime}+2 \omega_{0 y} u, \\
& \frac{1}{\rho_{\mathrm{e}}} \frac{\partial \rho^{\prime}}{\partial t}+\frac{\partial u}{\partial x}+\frac{\partial v}{\partial y}+\frac{\partial w}{\partial z}=\alpha\left(\gamma_{\mathrm{A}}-\gamma\right) w .
\end{aligned}
$$

$$
\frac{\partial \theta}{\partial t}=\gamma \cdot w
$$

Добавим уравнение состояния:

$$
p=\rho R T, \quad p^{\prime}=\rho_{\mathrm{e}} R \theta+R T_{\mathrm{e}} \rho^{\prime}
$$

Тогда система уравнений (12) - (14) запишется в виде

$$
\begin{aligned}
& \frac{\partial u}{\partial t}=-R \frac{\partial \theta}{\partial x}-\frac{R T_{\mathrm{e}}}{\rho_{\mathrm{e}}} \frac{\partial \rho^{\prime}}{\partial x}+2 w \omega_{0 z}-2 w \omega_{0 y} \\
& \frac{\partial v}{\partial t}=-R \frac{\partial \theta}{\partial y}-\frac{R T_{\mathrm{e}}}{\rho_{\mathrm{e}}} \frac{\partial \rho^{\prime}}{\partial y}-2 \omega_{0 z} u \\
& \frac{\partial w}{\partial t}=-R \frac{\partial \theta}{\partial z}+R \alpha\left(\gamma_{\mathrm{A}}-\gamma\right) \theta-\frac{R T_{\mathrm{e}}}{\rho_{\mathrm{e}}} \frac{\partial \rho^{\prime}}{\partial z}-(g-R \gamma) \frac{\rho^{\prime}}{\rho_{\mathrm{e}}}+2 \omega_{0 y} u
\end{aligned}
$$

Таким образом, мы получаем систему уравнений (12) - (20). Далее поступим также как и в [6], возьмем производную по координате $x$ от (18) и по координате $y$ от (19), производную по переменной $z$ от (20), сложим и получим

$$
\begin{aligned}
& \alpha\left(\gamma_{\mathrm{A}}-\gamma\right) \frac{\partial w}{\partial t}-\frac{1}{\rho_{\mathrm{e}}} \frac{\partial^{2} \rho^{\prime}}{\partial t^{2}}=R \alpha\left(\gamma_{\mathrm{A}}-\gamma\right) \frac{\partial \theta}{\partial z}-R \nabla^{2} \theta-\frac{R T_{\mathrm{e}}}{\rho_{\mathrm{e}}} \nabla^{2} \rho^{\prime}+2 \omega_{0 z} \Omega_{z}+2 \omega_{0 y} \Omega_{y^{-}} \\
& -\left[g+\alpha\left(\gamma_{\mathrm{A}}-\gamma\right) R T_{\mathrm{e}}-2 R \gamma\right] \frac{1}{\rho_{\mathrm{e}}} \frac{\partial \rho^{\prime}}{\partial z}-\alpha\left(\gamma_{\mathrm{A}}-\gamma\right)(g-R \gamma) \frac{\rho^{\prime}}{\rho_{\mathrm{e}}}
\end{aligned}
$$




$$
\begin{aligned}
& \alpha\left(\gamma_{\mathrm{A}}-\gamma\right) \frac{\partial^{2} w}{\partial t^{2}}-\frac{1}{\rho_{\mathrm{e}}} \frac{\partial^{3} \rho^{\prime}}{\partial t^{3}}=-R \nabla^{2} \frac{\partial \theta}{\partial t}-\frac{R T_{\mathrm{e}}}{\rho_{\mathrm{e}}} \nabla^{2} \frac{\partial \rho^{\prime}}{\partial t}+2 \omega_{0 z} \frac{\partial \Omega_{\mathrm{z}}}{\partial t}+2 \omega_{0 y} \frac{\partial \Omega_{y}}{\partial t}+ \\
& +R \alpha\left(\gamma_{\mathrm{A}}-\gamma\right) \frac{\partial \theta}{\partial t}+\frac{\alpha R \gamma\left(\gamma_{\mathrm{A}}-\gamma\right)}{\rho_{\mathrm{e}}} \frac{\partial \rho^{\prime}}{\partial t}-\left(g-2 R \gamma+\alpha R\left(\gamma_{\mathrm{A}}-\gamma\right) T_{\mathrm{e}}\right) \frac{1}{\rho_{\mathrm{e}}} \frac{\partial}{\partial z} \frac{\partial \rho^{\prime}}{\partial t} .
\end{aligned}
$$

Так как

$\frac{\partial \theta}{\partial t}=\gamma \cdot w, \quad \frac{\partial \nabla^{2} \theta}{\partial t}=\gamma \cdot \nabla^{2} w$,

To

$$
\begin{gathered}
\alpha\left(\gamma_{\mathrm{A}}-\gamma\right) \frac{\partial^{2} w}{\partial t^{2}}-\frac{1}{\rho_{\mathrm{e}}} \frac{\partial^{3} \rho^{\prime}}{\partial t^{3}}=R \alpha \gamma\left(\gamma_{\mathrm{A}}-\gamma\right) \frac{\partial w}{\partial z}-\gamma R \nabla^{2} w-\frac{R T_{\mathrm{e}}}{\rho_{\mathrm{e}}} \nabla^{2} \frac{\partial \rho^{\prime}}{\partial t}+\frac{\partial}{\partial t}\left(2 \omega_{0 z} \Omega_{z}+2 \omega_{0 y} \Omega_{y}\right)^{-} \\
-\left[g+\alpha\left(\gamma_{\mathrm{A}}-\gamma\right) R T_{\mathrm{e}}-2 R \gamma\right] \frac{1}{\rho_{\mathrm{e}}} \frac{\partial}{\partial z} \frac{\partial \rho^{\prime}}{\partial t}-\alpha\left(\gamma_{\mathrm{A}}-\gamma\right)(g-R \gamma) \frac{1}{\rho_{\mathrm{e}}} \frac{\partial \rho^{\prime}}{\partial t}
\end{gathered}
$$

Разделим это уравнение на два равенства:

$$
\begin{aligned}
& \frac{\partial^{2} \rho^{\prime}}{\partial t^{2}}=R T_{\mathrm{e}} \nabla^{2} \rho^{\prime}-\left[g+\alpha\left(\gamma_{\mathrm{A}}-\gamma\right) R T_{\mathrm{e}}-2 R \gamma\right] \frac{\partial \rho^{\prime}}{\partial z}+\alpha\left(\gamma_{\mathrm{A}}-\gamma\right)(g-R \gamma) \rho^{\prime} \\
& \frac{\partial^{2} w}{\partial t^{2}}=-\frac{R \gamma}{\alpha\left(\gamma_{\mathrm{A}}-\gamma\right)} \cdot \nabla^{2} w+R \gamma \frac{\partial w}{\partial z}+\frac{1}{\alpha\left(\gamma_{\mathrm{A}}-\gamma\right)} \frac{\partial}{\partial t} 2\left(\omega_{0 z} \Omega_{z}+\omega_{0 y} \Omega_{y}\right)
\end{aligned}
$$

где

$\Omega_{z}=\frac{\partial v}{\partial x}-\frac{\partial u}{\partial y}, \quad \Omega_{y}=\frac{\partial u}{\partial z}-\frac{\partial w}{\partial x}$

- проскции вихря скорости

Уравнение переноса вихря получается из уравнений движений (18) и (19) дифференцированием (18) по координате $y$, а (19) по координате $x$ и вычитанием полученных выражений:

$$
\frac{\partial \Omega_{z}}{\partial t}=-2 \omega_{0 z}\left[\alpha\left(\gamma_{\mathrm{A}}-\gamma\right) w-\frac{\partial w}{\partial z}-\frac{1}{\rho_{\mathrm{e}}} \frac{\partial \rho^{\prime}}{\partial t}\right]+2 \omega_{0 y} \frac{\partial w}{\partial y}
$$

Аналогично,

$$
\frac{\partial \Omega_{y}}{\partial t}=-R \alpha\left(\gamma_{\mathrm{A}}-\gamma\right) \frac{\partial \theta}{\partial x}+\left[g-\alpha\left(\gamma_{\mathrm{A}}-\gamma\right) R T_{\mathrm{e}}\right] \frac{1}{\rho_{\mathrm{e}}} \frac{\partial \rho^{\prime}}{\partial x}+2 \omega_{0 z} \frac{\partial v}{\partial z}-2 \omega_{0 y}\left[\alpha\left(\gamma_{\mathrm{A}}-\gamma\right) w-\frac{1}{\rho_{\mathrm{e}}} \frac{\partial \rho^{\prime}}{\partial t}-\frac{\partial v}{\partial y}\right] \text { (26) }
$$


Аналогично,

$$
\frac{\partial \Omega_{x}}{\partial t}=R \alpha\left(\gamma_{\mathrm{A}}-\gamma\right) \frac{\partial \theta}{\partial y}-\left[g-\alpha\left(\gamma_{\mathrm{A}}-\gamma\right) R T_{\mathrm{e}}\right] \frac{1}{\rho_{\mathrm{e}}} \frac{\partial \rho^{\prime}}{\partial y}+2 \omega_{0 y} \frac{\partial u}{\partial y}+2 \omega_{0 z} \frac{\partial u}{\partial z}
$$

Отсюда

$\frac{\partial^{2}}{\partial t^{2}} 2\left(\omega_{0 y} \Omega_{y}+\omega_{0 z} \Omega_{z}\right)=$

$=-\left[\left(2 \omega_{0 y}\right)^{2}+\left(2 \omega_{0 z}\right)^{2}\right]\left[\alpha\left(\gamma_{\mathrm{A}}-\gamma\right) \frac{\partial w}{\partial t}-\frac{1}{\rho_{\mathrm{e}}} \frac{\partial^{2} \rho^{\prime}}{\partial t^{2}}\right]+\left(4 \omega_{0 y} \frac{\partial}{\partial y}+4 \omega_{0 z} \frac{\partial}{\partial z}\right) \frac{\partial}{\partial t}\left(\omega_{0 y} v+\omega_{0 z} w_{j}\right)+$

$-2 \omega_{0 y} R \alpha \gamma\left(\gamma_{\mathrm{A}}-\gamma\right) \frac{\partial w}{\partial x}+2 \omega_{0 y}\left[g-\alpha\left(\gamma_{\mathrm{A}}-\gamma\right) R T_{\mathrm{e}}\right] \frac{1}{\rho_{\mathrm{e}}} \frac{\partial}{\partial x} \frac{\partial \rho^{\prime}}{\partial t}$.

Из уравнений (18) - (2\0) найдем

$$
\begin{aligned}
& \frac{\partial^{2}}{\partial t^{2}}\left(\omega_{0 y} v+\omega_{0 z} w\right)=\omega_{0 z} R \alpha\left(\gamma_{\mathrm{A}}-\gamma\right) \gamma w-R \gamma\left(\omega_{0 y} \frac{\partial}{\partial y}+\omega_{0 z} \frac{\partial}{\partial z}\right) w- \\
& -\frac{R T_{\mathrm{e}}}{\rho_{\mathrm{e}}}\left(\omega_{0 y} \frac{\partial}{\partial y}+\omega_{0 z} \frac{\partial}{\partial z}\right) \frac{\partial \rho^{\prime}}{\partial t}-\omega_{0 z}(g-R \gamma) \frac{1}{\rho_{\mathrm{e}}} \frac{\partial \rho^{\prime}}{\partial t}
\end{aligned}
$$

Отсюда

$$
\begin{aligned}
& \frac{\partial^{3}}{\partial t^{3}} 2\left(\omega_{0 y} \Omega_{y}+\omega_{0 z} \Omega_{z}\right)=-\left[\left(2 \omega_{0 y}\right)^{2}+\left(2 \omega_{0 z}\right)^{2}\right]\left[\alpha\left(\gamma_{\mathrm{A}}-\gamma\right) \frac{\partial^{2} w}{\partial t^{2}}-\frac{1}{\rho_{\mathrm{e}}} \frac{\partial^{3} \rho^{\prime}}{\partial t^{3}}\right]- \\
& -2 \omega_{0 y} R \alpha \gamma\left(\gamma_{\mathrm{A}}-\gamma\right) \frac{\partial}{\partial x} \frac{\partial w}{\partial t}+2 \omega_{0 y}\left[g-\alpha\left(\gamma_{\mathrm{A}}-\gamma\right) R T_{\mathrm{e}}\right] \frac{1}{\rho_{\mathrm{e}}} \frac{\partial}{\partial x} \frac{\partial^{2} \rho^{\prime}}{\partial t^{2}}+ \\
& +\left(4 \omega_{0 y} \frac{\partial}{\partial y}+4 \omega_{0 z} \frac{\partial}{\partial z}\right)\left\{\omega_{0 z} R \alpha\left(\gamma_{\mathrm{A}}-\gamma\right) \gamma w-R \gamma\left(\omega_{0 y} \frac{\partial}{\partial y}+\omega_{0 z} \frac{\partial}{\partial z}\right) w-\right. \\
& \left.-\frac{R T_{\mathrm{e}}}{\rho_{\mathrm{e}}}\left(\omega_{0 y} \frac{\partial}{\partial y}+\omega_{0 z} \frac{\partial}{\partial z}\right) \frac{\partial \rho^{\prime}}{\partial t}-\omega_{0 z}(g-R \gamma) \frac{1}{\rho_{\mathrm{e}}} \frac{\partial \rho^{\prime}}{\partial t}\right\}
\end{aligned}
$$

Таким образом, получаем систему уравнений

$$
\frac{\partial^{2} w}{\partial t^{2}}=-\frac{R \gamma}{\alpha\left(\gamma_{\mathrm{A}}-\gamma\right)} \cdot \nabla^{2} w+R \gamma \frac{\partial w}{\partial z}+\frac{1}{\alpha\left(\gamma_{\mathrm{A}}-\gamma\right)} \frac{\partial}{\partial t} 2\left(\omega_{0 z} \Omega_{z}+\omega_{0 y} \Omega_{y}\right)
$$




$$
\begin{aligned}
& \frac{\partial^{3}}{\partial t^{3}} 2\left(\omega_{0 y} \Omega_{y}+\omega_{0 z} \Omega_{z}\right)=-\left[\left(2 \omega_{0 y}\right)^{2}+\left(2 \omega_{0 z}\right)^{2}\right]\left[\alpha\left(\gamma_{\mathrm{A}}-\gamma\right) \frac{\partial^{2} w}{\partial t^{2}}-\frac{1}{\rho_{\mathrm{e}}} \frac{\partial^{3} \rho^{\prime}}{\partial t^{3}}\right]- \\
& -2 \omega_{0 y} R \alpha \gamma\left(\gamma_{\mathrm{A}}-\gamma\right) \frac{\partial}{\partial x} \frac{\partial w}{\partial t}+2 \omega_{0 y}\left[g-\alpha\left(\gamma_{\mathrm{A}}-\gamma\right) R T_{\mathrm{e}}\right] \frac{1}{\rho_{\mathrm{e}}} \frac{\partial}{\partial x} \frac{\partial^{2} \rho^{\prime}}{\partial t^{2}}+ \\
& +\left(4 \omega_{0 y} \frac{\partial}{\partial y}+4 \omega_{0 z} \frac{\partial}{\partial z}\right)\left\{\omega_{0 z} R \alpha\left(\gamma_{\mathrm{A}}-\gamma\right) \gamma w-R \gamma\left(\omega_{0 y} \frac{\partial}{\partial y}+\omega_{0 z} \frac{\partial}{\partial z}\right) w-\right. \\
& \left.-\frac{R T_{\mathrm{e}}}{\rho_{\mathrm{e}}}\left(\omega_{0 y} \frac{\partial}{\partial y}+\omega_{0 z} \frac{\partial}{\partial z}\right) \frac{\partial \rho^{\prime}}{\partial t}-\omega_{0 z}(g-R \gamma) \frac{1}{\rho_{\mathrm{e}}} \frac{\partial \rho^{\prime}}{\partial t}\right\} \\
& \frac{\partial^{2} \rho^{\prime}}{\partial t^{2}}=R T_{\mathrm{e}} \nabla^{2} \rho^{\prime}-\alpha R \gamma\left(\gamma_{\mathrm{A}}-\gamma\right) \rho^{\prime}+\left[\rho_{\mathrm{e}} g-2 R \gamma+\alpha R\left(\gamma_{\mathrm{A}}-\gamma\right) T_{\mathrm{e}}\right] \frac{\partial \rho^{\prime}}{\partial z}
\end{aligned}
$$

Будем искать решение полученной системы в виде:

$$
2\left(\omega_{0 y} \Omega_{y}+\omega_{0 z} \Omega_{z}\right)=\Omega_{0}^{2} e^{-i \omega t}, \quad w=W e^{-i \omega t}, \quad \rho^{\prime}=D e^{-i \omega t} .
$$

Из уравнений получим

$$
\begin{aligned}
& -\omega^{2} W+\frac{i \omega}{\alpha\left(\gamma_{\mathrm{A}}-\gamma\right)} \Omega_{0}^{2}=-\frac{R \gamma}{\alpha\left(\gamma_{\mathrm{A}}-\gamma\right)} \cdot \nabla^{2} W+R \gamma \frac{\partial W}{\partial z} \\
& -\omega^{2} D=R T_{\mathrm{e}} \nabla^{2} D-\alpha R \gamma\left(\gamma_{\mathrm{A}}-\gamma\right) D+\left[\rho_{\mathrm{e}} g-2 R \gamma+\alpha R\left(\gamma_{\mathrm{A}}-\gamma\right) T_{\mathrm{e}}\right] \frac{\partial D}{\partial z}
\end{aligned}
$$

$i \omega^{3} \Omega_{0}^{2}-\left[\left(2 \omega_{0 y}\right)^{2}+\left(2 \omega_{0 z}\right)^{2}\right] \alpha\left(\gamma_{\mathrm{A}}-\gamma\right) \omega^{2} W-\frac{1}{\rho_{\mathrm{e}}}\left[\left(2 \omega_{0 y}\right)^{2}+\left(2 \omega_{0 z}\right)^{2}\right] i \omega^{3} D=$

$=2 i \omega \cdot \omega_{0 y} R \alpha \gamma\left(\gamma_{\mathrm{A}}-\gamma\right) \frac{\partial W}{\partial x}-2 \omega^{2} \cdot \omega_{0 y}\left[g-\alpha\left(\gamma_{\mathrm{A}}-\gamma\right) R T_{\mathrm{e}}\right] \frac{1}{\rho_{\mathrm{e}}} \frac{\partial D}{\partial x}+$

$+4 \omega_{0 z} R \alpha\left(\gamma_{\mathrm{A}}-\gamma\right) \gamma\left(\omega_{0 y} \frac{\partial}{\partial y}+\omega_{0 z} \frac{\partial}{\partial z}\right) W-4 R \gamma\left(\omega_{0 y} \frac{\partial}{\partial y}+\omega_{0 z} \frac{\partial}{\partial z}\right)^{2} W-$

$$
+4 i \omega \cdot \frac{R T_{\mathrm{e}}}{\rho_{\mathrm{e}}}\left(\omega_{0 y} \frac{\partial}{\partial y}+\omega_{0 z} \frac{\partial}{\partial z}\right)^{2} D+4 i \omega \cdot \omega_{0 z} \frac{g-R \gamma}{\rho_{\mathrm{e}}}\left(\omega_{0 y} \frac{\partial}{\partial y}+\omega_{0 z} \frac{\partial}{\partial z}\right) D
$$

Найдем решение уравнения (36)

$$
\nabla^{2} D+\frac{g-2 R \gamma+\alpha\left(\gamma_{\mathrm{A}}-\gamma\right) R T_{\mathrm{e}}}{R T_{\mathrm{e}}} \frac{\partial D}{\partial z}+\frac{\omega^{2}-\alpha R \gamma\left(\gamma_{\mathrm{A}}-\gamma\right)}{R T_{\mathrm{e}}} D=0 .
$$


Решение ищем в виде:

$D=D_{0}(z) e^{i\left(k_{1} x+k_{2} y\right)}, \quad 2\left(\omega_{0 y} \Omega_{y}+\omega_{0 z} \Omega_{z}\right)=\tilde{\Omega}_{0}^{2}(z) e^{i\left(k_{1} x+k_{2} y-\omega t\right)}$,

$w=W_{0}(z) e^{i\left(k_{1} x+k_{2} y-\omega t\right)}$.

Введем обозначение

$b=g-2 R \gamma+\alpha R\left(\gamma_{\mathrm{A}}-\gamma\right) T_{\mathrm{e}}, \quad \tilde{\omega}=\alpha R \gamma\left(\gamma_{\mathrm{A}}-\gamma\right)$

Тогда

$\frac{\partial^{2} D_{0}(z)}{\partial z^{2}}+\frac{b}{R T_{\mathrm{e}}} \frac{\partial D_{0}(z)}{\partial z}+\left(\frac{\omega^{2}-\tilde{\omega}^{2}}{R T_{\mathrm{e}}}+k^{2}\right) D_{0}(z)=0$.

Перейдем к новой функции

$D_{0}=e^{\int \tilde{D}_{0} \mathrm{~d} z}, \quad \frac{\partial D_{0}}{\partial z}=\tilde{D}_{0} e^{\int \tilde{D}_{0} \mathrm{~d} z}, \quad \frac{\partial^{2} D_{0}}{\partial z^{2}}=\tilde{D}_{0}^{2} e^{\int \tilde{D}_{0} \mathrm{~d} z}+\frac{\partial \tilde{D}_{0}}{\partial z} e^{\int \tilde{D}_{0} \mathrm{~d} z}$

Тогда

$\frac{\partial \tilde{D}_{0}}{\partial z}+\tilde{D}_{0}^{2}+\frac{b}{R T_{\mathrm{e}}} \tilde{D}_{0}+\left(\frac{\omega^{2}-\tilde{\omega}^{2}}{R T_{\mathrm{e}}}+k^{2}\right)=0$.

Отсюда

$-\frac{\partial \tilde{D}_{0}}{\partial z}=\left(\tilde{D}_{0}-\tilde{D}_{1}(z)\right)\left(\tilde{D}_{0}-\tilde{D}_{2}(z)\right)$

$\int \frac{\partial \tilde{D}_{0}}{\left(\tilde{D}_{0}-\tilde{D}_{1}(z)\right)\left(\tilde{D}_{0}-\tilde{D}_{2}(z)\right)}=-z+$ const.

Будем считать, что мы нашли решение этого уравнения и функция $\widetilde{D}_{0}=\widetilde{D}_{0}(z)$ нам известна. Тогда

$D=D_{0}(z) e^{k_{1} x+k_{2} y}$

Из (35) найдем.

$$
i \omega \Omega_{0}^{2}=\omega^{2} W-R \gamma \cdot \nabla^{2} W+\alpha\left(\gamma_{\mathrm{A}}-\gamma\right) R \gamma \frac{\partial W}{\partial z} .
$$


Подставим полученное выражение в (36):

$$
\begin{aligned}
& \omega^{4} W_{0}-\omega^{2} R \gamma \cdot \frac{\partial^{2} W_{0}}{\partial z^{2}}+\omega^{2} \alpha\left(\gamma_{\mathrm{A}}-\gamma\right) R \gamma \frac{\partial W_{0}}{\partial z}-\omega^{2}\left[\left(2 \omega_{0 y}\right)^{2}+\left(2 \omega_{0 z}\right)^{2}\right] \alpha\left(\gamma_{\mathrm{A}}-\gamma\right) W_{0^{-}} \\
& -4 \omega_{0 z} R \alpha\left(\gamma_{\mathrm{A}}-\gamma\right) \gamma\left(i k_{2} \omega_{0 y} W_{0}+\omega_{0 z} \frac{\partial W_{0}}{\partial z}\right)+4 R \gamma\left(i k_{2} \omega_{0 y} W_{0}+\omega_{0 z} \frac{\partial W_{0}}{\partial z}\right)^{2}+ \\
& +2 k_{1} \omega \cdot \omega_{0 y} R \alpha \gamma\left(\gamma_{\mathrm{A}}-\gamma\right) W_{0}= \\
& =\frac{1}{\rho_{\mathrm{e}}}\left[\left(2 \omega_{0 y}\right)^{2}+\left(2 \omega_{0 z}\right)^{2}\right] i \omega^{3} D_{0}-2 \omega^{2} \cdot \omega_{0 y}\left[g-\frac{\alpha R\left(\gamma_{\mathrm{A}}-\gamma\right) T_{\mathrm{e}}}{\rho_{\mathrm{e}}}\right] i k_{1} D_{0}+ \\
& +4 i \omega \cdot \frac{R T_{\mathrm{e}}}{\rho_{\mathrm{e}}}\left(i k_{2} \omega_{0 y} D_{0}+\omega_{0 z} \frac{\partial D_{0}}{\partial z}\right)^{2}+4 i \omega \cdot \omega_{0 z}\left(g-\frac{R \gamma}{\rho_{\mathrm{e}}}\right)\left(i k_{2} \omega_{0 y} D_{0}+\omega_{0 z} \frac{\partial D_{0}}{\partial z}\right)
\end{aligned}
$$

Найдем решение при условии $D_{0}=0$ и $k_{2}=0$. Получим

$$
\begin{aligned}
& \omega^{4} W_{0}-\omega^{2} R \gamma \cdot \frac{\partial^{2} W_{0}}{\partial z^{2}}+\omega^{2} \alpha\left(\gamma_{A^{-}} \gamma\right) R \gamma \frac{\partial W_{0}}{\partial z}-\omega^{2}\left[\left(2 \omega_{0 y}\right)^{2}+\left(2 \omega_{0 z}\right)^{2}\right] \alpha\left(\gamma_{A^{-}} \gamma\right) W_{0^{-}} \\
& -\left(2 \omega_{0 z}\right)^{2} R \alpha\left(\gamma_{\mathrm{A}}-\gamma\right) \gamma \frac{\partial W_{0}}{\partial z}+\left(2 \omega_{0 z}\right)^{2} R \gamma\left(\frac{\partial W_{0}}{\partial z}\right)^{2}+2 k_{1} \omega \cdot \omega_{0 y} R \alpha \gamma\left(\gamma_{\mathrm{A}}-\gamma\right) W_{0}=0
\end{aligned}
$$

Решение ищем в виде $W_{0}=W_{00} e^{k_{z z} z}$. Тогда получим ducnepcuонное coomномение в виде:

$$
\begin{aligned}
& \omega^{4}-\left\{R \gamma k_{z}^{2}-\alpha\left(\gamma_{\mathrm{A}}-\gamma\right) R \gamma k_{z}+\left[\left(2 \omega_{0 y}\right)^{2}+\left(2 \omega_{0 z}\right)^{2}\right] \alpha\left(\gamma_{\mathrm{A}}-\gamma\right)\right\} \omega^{2}+ \\
& +2 \omega_{0 y} R \alpha \gamma\left(\gamma_{\mathrm{A}}-\gamma\right) \cdot k_{1} \omega-\left(2 \omega_{0 z}\right)^{2} R \alpha\left(\gamma_{\mathrm{A}}-\gamma\right) \gamma k_{z}+\left(2 \omega_{0 z}\right)^{2} R \gamma k_{z}^{2}=0 .
\end{aligned}
$$

\section{Результаты исследования и их обсукдение}

Получилось сложное уравнение 4-го порядка. Рассмотрим предельные случаи: высокочастотные и низкочастотные колебания. В случае высокочастотных колебаний можно записать:

$$
\begin{aligned}
& \omega^{4}-\left(2 \omega_{0 z}\right)^{2} R \alpha\left(\gamma_{\mathrm{A}}-\gamma\right) \gamma k_{z}+\left(2 \omega_{0 z}\right)^{2} R \gamma k_{z}^{2}=0 \\
& \omega=(1 \pm i) \sqrt{\omega_{0 z}}\left(R \gamma k_{z}\right)^{1 / 4}=\omega_{\mathrm{r}} \pm i \omega_{\mathrm{i}} \\
& \omega_{\mathrm{r}}=\sqrt{\omega_{0 z}}\left(R \gamma k_{z}\right)^{1 / 4}
\end{aligned}
$$


Для низкочастотных колебаний получим:

$$
\begin{aligned}
& -\left\{R \gamma k_{z}^{2}-\alpha\left(\gamma_{\mathrm{A}}-\gamma\right) R \gamma k_{z}+\left[\left(2 \omega_{0 y}\right)^{2}+\left(2 \omega_{0 z}\right)^{2}\right] \alpha\left(\gamma_{\mathrm{A}}-\gamma\right)\right\} \omega^{2}+2 \omega_{0 y} R \alpha \gamma\left(\gamma_{\mathrm{A}}-\gamma\right) \cdot k_{1} \omega=0, \\
& \omega=\frac{2 \omega_{0 y} R \gamma \cdot k_{1}}{R \gamma k_{z}\left[\frac{k_{z}}{\alpha\left(\gamma_{\mathrm{A}}-\gamma\right)}-1\right]+\left(2 \omega_{0 y}\right)^{2}+\left(2 \omega_{0 z}\right)^{2}}
\end{aligned}
$$

Из формулы (50) видно, что чем больше длина волны, тем меньше частота колебаний волны.

Для фазовой скорости волны получим выражение:

$$
c_{1}=\frac{\omega}{k_{1}}=\frac{2 \omega_{0 y} R \gamma}{R \gamma k_{z}\left[\frac{k_{z}}{\alpha\left(\gamma_{\mathrm{A}}-\gamma\right)}-1\right]+\left(2 \omega_{0 y}\right)^{2}+\left(2 \omega_{0 z}\right)^{2}} .
$$

Из формулы (51) видно, что чем ближе к полюсу, тем скорость волны меньше

\section{Выводы}

Таким образом, рассмотрев систему уравнений, описывающих динамику внутренних гравитационных волн в атмосфере, после линеаризации мы получили дисперсионное соотношение. Система уравнений решалась в приближении f-плоскости, т.е. параметр Кориолиса считался постоянным. При этом считалось, что плотность воздуха в состоянии статики атмосферы подчиняется барометрическому закону. В пределах тропосферы такое допущение является приемлемым. Кроме того, вертикальный градиент температуры в состоянии статики атмосферы (невозмушенное состояние) также считается постоянным. Аналитическое решение было получено для случая невязкого и нетеплопроводного воздуха. Мы не рассматривали проблему допустимости процедуры линеаризации для волн планетарного масштаба. Предполагалось, что для указанных волн данная процедура имеет место.

При сделанных выше допущениях было получено выражение для скорости планетарной волны, из которой следует, что при приближении к полюсу, тее. с увеличением широты, скорость распространения волны уменьшается.

\section{Работа выполнена под научным руководством профессора Р.Г. Закиняна.}




\section{Библиографический список}

1. Гилл А. Динамика атмосферы и океана. М.: Мир, 1986, Т. 1, 399 с.; Т. 2, 416 c.

2. Матвеев Л.Т. Теория общей циркуляции атмосферы и климата Земли. Л.: Гидрометеоиздат, 1991, 295 с.

3. Шакина Н.П. Лекции по динамической метеорологии. М. ТРИАДА ЛТД, 2013. $160 \mathrm{c.}$

4. Andrews D.G. An Introduction to Atmospheric Physics. Second edition. Cambridge University Press, 2010, p. 237.

5. Cushman-Roisin B., Beckers J-M. Introduction to Geophysical Fluid Dynamics. Second edition. International geophysics series, vol. 101. Elsevier, 2011, p. 828.

6. Holton J.R. An Introduction to Dynamic Meteorology. Forth edition Elsevier, 2004, p. 540.

7. Marshall J., Plumb R.A. Atmosphere, Ocean, and Climate Dynamics. International geophysics series, vol. 93. Elsevier, 2008, p. 324.

8. Nappo C.J. An Introduction to Atmospheric Gravity Waves. International geophysics series, vol. 85. Elsevier, 2002, p. 279

9. Pedlosky J. Waves in the Ocean and Atmosphere. Springer-Verlag Berlin Heidelberg, 2003, p 260.

10. Zdunkowski W., Bott A. Dynamics of the Atmosphere. Cambridge University Press, 2003, p. 719.

\section{References}

1. Gill A. Dynamics of the atmosphere and the ocean. M.: Mir, 1986, T. 1, 399 p.; T. 2, $416 \mathrm{~s}$.

2. Matveyev L.T. The theory of the general circulation of the atmosphere and climate of the Earth. L.: Hydrometeoizdat, 1991, $295 p$

3. Shakina N.P. Lectures on dynamic meteorology. M.: TRIADA LTD 2013. $160 \mathrm{p}$.

4. Andrews D.G. An Introduction to Atmospheric Physics. Second edition. Cambridge University Press, 2010, p. 237.

5. Cushman-Roisin B., Beckers J-M. Introduction to Geophysical Fluid Dynamics. Second edition. International geophysics series, vol. 101. Elsevier, 2011, p. 828

6. Holton J.R. An Introduction to Dynamic Meteorology. Forth edition. Elsevier, 2004, p. 540.

7. Marshall J., Plumb R.A. Atmosphere, Ocean, and Climate Dynamics. International geophysics series, vol. 93. Elsevier, 2008, p. 324.

8. Nappo C.J. An Introduction to Atmospheric Gravity Waves. International geophysics series, vol. 85. Elsevier, 2002, p. 279

9. Pedlosky J. Waves in the Ocean and Atmosphere. Springer-Verlag Berlin Heidelberg, 2003, p. 260.

10. Zdunkowski W., Bott A. Dynamics of the Atmosphere. Cambridge University Press, 2003, p. 719. 
Рукопись поступила в редакцию 10.12.2018 г.

Принята к публикации 25.02.2019 г.

\section{O6 авторах}

Набродова Е.Г., аспирант кафедры общей и теоретической физики СевероКавказского федерального университета Телефон: 8-918-75348-97 E-mail: katy-popova1991@yandecs.ru

Шмигельский В.А., аспирант кафедры общей и теоретической ффизики СевероКавказского федерального университета Телефон 8(906) 470-57-25 E-mail: shmigelskiy.92777@mail.ru ResearcherID is Q-3869-2018

Яновская О.С., аспирант кафедры общей и теоретической физики СевероКавказского федерального университета Телефон: 8-905-41514-14 E-mail: olenka_yan@mail.ru

Сурнева О.Б., аспирант кафредры общей и теоретической физики СевероКавказского федерального университета Телефон: 8-918-74492-18 E-mail: surnevao@mail.ru

\section{About the authors}

Nabrodova E.G., Post Post-Graduate Student, Department of General and Theoretical Physics, North-Caucasian Federal University.

Phone: 8-918-753-48-97 E-mail: katy-popova1991@yandecs.ru

Shmigelsky V., Post-Graduate Student, Department of General and Theoretical Physics, North-Caucasian Federal University

Phone 8 (906) 470-57-25

E-mail: shmigelskiy.92777@mail.ru ResearcherID is: Q-3869-2018

Yanovskaya O.S, Post-Graduate Student, Department of General and Theoretical Physics, North-Caucasian Federal University.

Phone: 8-905-415-14-14 E-mail: olenka_yan@mail.ru.

Surneva O.B., Post-Graduate Student, Department of General and Theoretical Physics, North-Caucasian Federal University

Phone: 8-918-744-92-18. E-mail: surnevao@mail.ru 